\title{
Estudo RADAR - Risco Aumentado de Diabetes em Amarante
}

Teresa Valente,* Lúcia Azevedo**

\section{RESUMO}

Objectivos: Caracterizar os utentes dos Cuidados de Saúde Primários quanto ao risco de virem a desenvolver Diabetes Mellitus tipo 2 (DM2).

Tipo de estudo: Observacional, descritivo e transversal.

Local: Centro de Saúde (CS) de Amarante.

População: Utentes inscritos no CS de Amarante com idades compreendidas entre os 18 e os 79 anos ( $\mathrm{N}=28523)$.

Métodos: Foi aleatorizada uma amostra de 500 utentes. Para determinar o risco de desenvolver DM2 foi usado o Finnish Diabetes Risk Score (FINDRISC) que considera oito fatores de risco da doença para calcular o risco total. As variáveis em estudo foram o género e os fatores de risco de DM2 incluídos no FINDRISC. Foi realizada a análise estatística descritiva e inferencial dos dados.

Resultados: Aplicando o questionário FINDRISC, 12,8\% dos indivíduos apresentaram um risco elevado (pontuação $\geq 15$ ) de desenvolver DM2 nos próximos 10 anos. Em relação aos fatores de risco de DM2 avaliados, verificou-se que $18 \%$ apresentava IMC $\geq 30 \mathrm{~kg} / \mathrm{m}^{2}$, $25 \%$ não comia frutas e vegetais diariamente, $29 \%$ tomava medicação para a hipertensão arterial, $42 \%$ apresentava perímetro abdominal compatível com obesidade central, $45 \%$ tinha pelo menos um familiar de $1 .^{\circ}$ ou $2 .^{\circ}$ grau com diagnóstico de DM2 e $45 \%$ não praticava atividade física regularmente.

Conclusões: Este estudo sugere que cerca de um em cada oito utentes observados ao nível dos CSP apresentam um risco elevado (FINDRISC $\geq 15$ ) de desenvolver diabetes nos próximos 10 anos. Os resultados encontrados reforçam a importância da utilização de uma escala de avaliação de risco, simples e de fácil aplicação, que permita ao médico de família intervir precocemente alertando o doente para o seu risco e promovendo estilos de vida saudável.

Palavras-Chave: Diabetes Mellitus Tipo 2; Prevalência; Avaliação de Risco; Cuidados de Saúde Primários.

\section{INTRODUÇÃO}

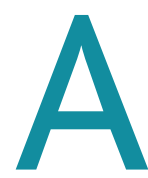
diabetes mellitus tipo 2 (DM2) é uma doença crónica associada a complicações bem conhecidas e com impacto negativo significativo na qualidade de vida dos doentes e das suas famílias. Algumas destas complicações podem constituir uma séria ameaça à sobrevivência dos doentes. Para além de ser uma importante causa de morbimortalidade, implica elevados gastos em saúde. Em 2008, os custos diretos com a doença, em Portugal, ascenderam aos 900-1100 milhões de euros, o que representou $7 \%$ da despesa em saúde para o mesmo ano. ${ }^{1,2}$

$\mathrm{O}$ aumento da prevalência de DM2 tem sido identificado como um importante problema de Saúde Pública. ${ }^{3}$

*Interna do $3 .^{\circ}$ ano de Medicina Geral e Familiar. UCSP Amadeo Souza Cardoso (Centro de Saúde de Amarante).

**Interna do $1 .^{\circ}$ ano de Medicina Geral e Familiar. UCSP Amadeo Souza Cardoso (Centro de Saúde de Amarante).
Em 2000 estimava-se que o número de pessoas com DM2, em todo o mundo, era de 171 milhões. Para 2030 prevê-se que esse número mais do que duplique. ${ }^{4}$ Em Portugal, segundo o Relatório Anual do Observatório Nacional da Diabetes, em 2008 a prevalência de DM foi de $11,7 \%$ e a de PréDiabetes de $23,2 \%$ da população portuguesa, com idades compreendidas entre os 20 e os 79 anos. $^{2}$

Assim, é reconhecida a necessidade de desenvolver estratégias de prevenção primária da DM2 que permitam, através da redução dos factores de risco modificáveis associados à doença, reduzir a prevalência da mesma. ${ }^{3,5,6}$ Para tal é fundamental identificar os indivíduos com risco acrescido de DM2 quando ainda se encontram normoglicémicos, intervindo no sentido de prevenir a evolução para Pré-diabetes e, posteriormente, Diabetes. ${ }^{3,5,6}$

Nesse sentido têm sido desenvolvidas várias escalas com o objetivo de estratificar o risco de vir a desenvolver DM2. ${ }^{3}$ São disso exemplo o «Diabetes Risk Test» da Associação 
Americana de Diabetes e o «Finnish Diabetes Risk Score» (FINDRISC) desenvolvido na Finlândia e validado para a língua inglesa. O FINDRISC demonstrou ser um método simples, rápido, pouco dispendioso e não invasivo para determinar o risco de desenvolver DM2 nos próximos 10 anos. ${ }^{7}$ Também tem demonstrado ser útil para identificar indivíduos com Diabetes não diagnosticada e Pré-Diabetes. ${ }^{8-9}$

As escalas de estratificação do risco de DM2, ao possibilitarem a identificação dos indivíduos com risco acrescido de desenvolver a doença, permitem que as estratégias de prevenção primária sejam direccionadas ao grupo de indivíduos que delas realmente beneficiam..,5,6 Vários ensaios clínicos têm demonstrado que os indivíduos com risco acrescido de diabetes podem reduzir significativamente o seu risco e protelar o início da doença adoptando uma dieta saudável, aumentando o nível de actividade física e mantendo ou reduzindo o seu IMC..$^{10-15}$

A Sociedade Europeia de Cardiologia e a Associação Europeia para o Estudo da Diabetes recomendam, nas Guidelines da Diabetes, Pré-Diabetes e Doenças Cardiovasculares, que a avaliação do risco de desenvolver DM2, usando as escalas disponíveis, deve fazer parte da rotina dos cuidados de saúde (nível de evidência A e classe de recomendação II) e que o rastreio do potencial de diabetes pode ser realizado de forma eficiente usando um score de risco não invasivo combinado, subsequentemente, com uma prova da tolerância à glicose oral (PTGO) naqueles com score de risco elevado (nível de evidência A e classe de recomendação I). ${ }^{16}$

O Programa Nacional de Prevenção e Controlo de Diabetes de 2008 contempla, como estratégia de intervenção, a identificação de grupos de risco acrescido de desenvolvimento de diabetes, através do preenchimento do Questionário de Avaliação do Risco de Diabetes Mellitus Tipo 2, a ser aplicado pelos profissionais de saúde. ${ }^{17}$ Embora esta versão traduzida do FINDRISC conste no Programa Nacional de Prevenção e Controlo de Diabetes e esteja disponível no programa informático $S A P E^{\circledR}$, ainda não está validada para a população portuguesa. Contudo, a Sociedade Portuguesa de Diabetologia, em colaboração com a Associação Protetora dos Diabéticos de Portugal, a Faculdade de Medicina de Coimbra e a Direção-Geral de Saúde pretendem, até Novembro de 2013, desenvolver o projeto de «Validação da Escala de Risco de Desenvolver Diabetes para a População Portuguesa - FINDRISC Portugal». ${ }^{18}$

Estudos realizados na Europa aplicando o questionário FINDRISC obtiveram valores médios de risco de 9,1 (Finlândia) e 10,7 (Espanha). ${ }^{8,19}$ Nos estudos de Makrilakis et al (Grécia) e Salinero-Fort et al (Espanha), 19,1\% e 19,5\% dos participantes apresentaram FINDRISC igual ou superior a 15 , respetivamente..$^{19,20}$

Este estudo teve como objectivo caracterizar os utentes dos Cuidados de Saúde Primários quanto ao risco de virem a desenvolver Diabetes Mellitus tipo 2 através da aplicação do questionário FINDRISC.

\section{MÉTODOS}

Tipo de estudo: Observacional, analítico transversal

Local do estudo: Centro de Saúde de Amarante

População do estudo: Utentes inscritos nas Unidades de Saúde do CS de Amarante (duas Unidades de Cuidados Personalizados, uma Unidade de Saúde Familiar e uma Unidade de utentes sem médico de família atribuído) com idades compreendidas entre os 18 e os 79 anos ( $\mathrm{N}=28523)$.

Critérios de exclusão: Pessoas com diabetes, grávidas, pessoas acamadas, internadas ou a viver em instituições não se podendo deslocar ao CS, pessoas afásicas, surdas ou com doença mental incapacitante ou com dificuldades de compreensão da língua portuguesa.

Caracterização da amostra e técnica de amostragem: Com base no estudo de Salinero-Fort et al definiu-se como prevalência esperada de FINDRISC $\geq 15$ o valor $19 \%$. Partindo deste valor e para uma população de 28523 utentes, um nível de precisão de $5 \%$ e um intervalo de confiança de 95\%, calculou-se, através do programa EpiInfo ${ }^{\circledR} 3.4 .3$, uma dimensão amostral de 235 indivíduos. Prevendo-se $50 \%$ de perdas por recusa na participação ou ausência de contactos atualizados, aumentou-se a dimensão da amostra para 470, tendo sido arredondado esse valor para 500 utentes. Através do programa «Gerador de amostras aleatórias para centros de saúde portugueses v 1.0», efetuou-se uma amostragem aleatória simples, estratificada por sexo e idade.

Variáveis em estudo: Foram estudadas a variável género e as variáveis incluídas no questionário FINDRISC-idade, peso, altura, índice de massa corporal (IMC), perímetro abdominal, prática de atividade física, alimentação com vegetais e fruta, história pessoal de Hipertensão Arterial, história pessoal de hiperglicemia, história familiar de diabetes - e a pontuação FINDRISC. O questionário FINDRISC é composto por 8 perguntas, o risco total é calculado somando o valor dos parâmetros individuais e varia entre 0 e 24. O risco individual é expresso em probabilidade de vir a desenvolver DM2 nos próximos 10 anos (Anexo 1).

Métodos de recrutamento de participantes: Os utentes aleatorizados foram convidados a participar no estudo através de uma carta de motivação, explicando o objectivo do estudo e convidando a pessoa a deslocar-se ao 
CS para uma entrevista individual com um dos investigadores, com dia e hora marcados. Nessa carta era disponibilizado o contacto telefónico do investigador para o caso do participante desejar marcar para outro dia e hora.

Plano de minimização de viéses: Para aumentar a taxa de resposta os utentes foram contactados por telefone na véspera do dia marcado com o objetivo de confirmar a participação. Nos casos em que foi demonstrado interesse em participar mas noutro dia, foi feita nova marcação de acordo com a disponibilidade do utente. Nos casos em que a carta foi devolvida, o utente foi contactado por telefone tendo sido efetuadas um máximo de três tentativas, em dias e horas diferentes. Foi elaborado um texto modelo para o contacto telefónico. Para uniformizar critérios inter-observador as primeiras 10 entrevistas foram realizadas pelos investigadores a pares definindo-se como proceder às medições directas. $\mathrm{O}$ peso e a altura foram medidos sem sapatos e acessórios e o perímetro abdominal avaliado ao nível da linha média entre a última costela e a crista ilíaca.

Métodos de recolha de informação: Os dados foram recolhidos através do Questionário de Avaliação de Risco de DM2 (FINDRISC), preenchido por entrevista pessoal e observação direta (peso, altura e perímetro abdominal) após explicação detalhada do estudo e assinado o consentimento informado pelo investigador e pelo participante.

O estudo foi aprovado pela Comissão de Ética da Administração Regional de Saúde do Norte.

Tratamento dos dados: Os dados foram codificados e registados em base de dados informática (Microsof Excel ${ }^{\circledR}$ ). Para o tratamento estatístico, utilizou-se o programa SPSS ${ }^{\circledR}$ v. 17.0. Realizou-se uma análise descritiva de cada uma das variáveis do estudo, as variáveis quantitativas expressaramse em medidas de tendência central e de dispersão e as qualitativas em frequências absolutas e relativas. Procedeu-se à divisão da amostra entre utentes com pontuação de FINDRISC $\geq 15 \mathrm{e}<15$. Foi usado o teste $t$ de Student para comparação de médias e o teste de qui-quadrado ou o teste de Fisher para a comparação de proporções. Em todos os casos o nível de significância adoptado foi de 0,05 .

\section{RESULTADOS}

Participaram no estudo 203 utentes, o que corresponde a uma taxa de resposta de 40,6\%. Em relação aos não respondentes (Quadro I), 55\% eram do sexo masculino e maioritariamente pertenciam à faixa etária entre os $28 \mathrm{e}$ os 38 anos.

A média de idades dos participantes no estudo foi de 45 anos $(D P=15)$ e $62 \%$ eram do género feminino. Relativa- mente aos fatores de risco de DM2 considerados no questionário FINDRISC (Quadro II), constatou-se que $17 \%$ dos participantes eram obesos, $42 \%$ apresentavam valores de perímetro abdominal definidores de obesidade central e $45 \%$ tinham história familiar de DM2. Quanto aos fatores de risco relacionados com estilos de vida verificou-se que $25 \%$ dos participantes não ingeria frutas e legumes diariamente e $45 \%$ não praticava atividade física regularmente.

QUADRO I. Caracterização das não respostas

\begin{tabular}{lcc} 
Motivo & $\mathbf{n}$ & $\mathbf{\%}$ \\
\hline Não contactáveis & 138 & 46,5 \\
\hline Contactados e recusaram & 45 & 15,2 \\
\hline Faltaram à marcação & 78 & 26,3 \\
\hline Não residentes & 36 & 12,1 \\
\hline
\end{tabular}

QUADRO II. Caracterização da amostra quanto às variáveis do questionário FINDRISC $(n=203)$

\begin{tabular}{lll} 
Variável & $\mathbf{n}$ & $\%$ \\
\hline Idade (anos) & & \\
$\quad 45-54$ & 43 & $21,2 \%$ \\
$\quad 55-64$ & 40 & $10,3 \%$ \\
$\quad 21$ & $10,3 \%$ \\
\hline IMC (Kg/m2) & & \\
$\quad 25-30$ & 94 & $46,3 \%$ \\
$\quad>30$ & 35 & $17,2 \%$ \\
\hline Perímetro Abdominal (cm) & & \\
$\quad$ H 94-102, M 80-88 & 63 & $31,0 \%$ \\
$\quad$ H > 102, M > 80 & 86 & $42,4 \%$ \\
\hline Sem actividade física regular & 92 & $45,3 \%$ \\
\hline HTA medicada & 58 & $28,6 \%$ \\
\hline Sem ingestão regular de legumes & 51 & $25,1 \%$ \\
\hline Antecedentes de hiperglicemia & 27 & $13,3 \%$ \\
\hline História Familiar de Diabetes & & \\
$\quad$ Sim, avós, tios, primos $1 .^{\circ}$ grau & 37 & $18,2 \%$ \\
$\quad$ Sim, pais, irmãos e filhos & 54 & $26,6 \%$ \\
\hline
\end{tabular}

Legenda: IMC - índice de massa corporal, $\mathrm{H}$ - Homens, $\mathrm{M}$ - Mulheres, HTA - Hipertensão Arterial 
A média da pontuação total obtida no questionário FINDRISC foi de 9,3 (IC95\%: 4,5-14,3) e 12,8\% (IC95\%: 7,8\% - 17,8\%) dos participantes apresentaram alto risco de desenvolver DM2 nos próximos 10 anos (FINDRISC $\geq 15$ ). Considerando apenas os participantes com idade superior a 45 anos, $22 \%$ apresentaram alto risco de desenvolver a doença (FINDRISC $\geq 15$ ).

A estratificação do risco dos participantes no estudo desenvolverem DM2 nos próximos 10 anos, segundo o questionário FINDRISC, encontra-se representada no Gráfico 1. Considerando como ponto de corte a pontuação FINDRISC $\geq 15$ usado nos estudos grego e espanhol para definir os indivíduos com risco elevado de desenvolver DM2, procedeu-se à análise comparativa dos dois grupos (Quadro IV). ${ }^{19-20}$

\section{DISCUSSÃO}

Os resultados obtidos revelaram que, ao nível dos cuidados de saúde primários, cerca de $13 \%$ dos utentes apresentam um alto risco de desenvolver DM2 nos próximos 10 anos. Esta percentagem foi inferior à obtida noutros estudos realizados na Europa, usando a mesma escala de risco. Nos estudos de Makrilakis etale de Salinero-Fort et al 19,1\% e $19,5 \%$ dos participantes, res-

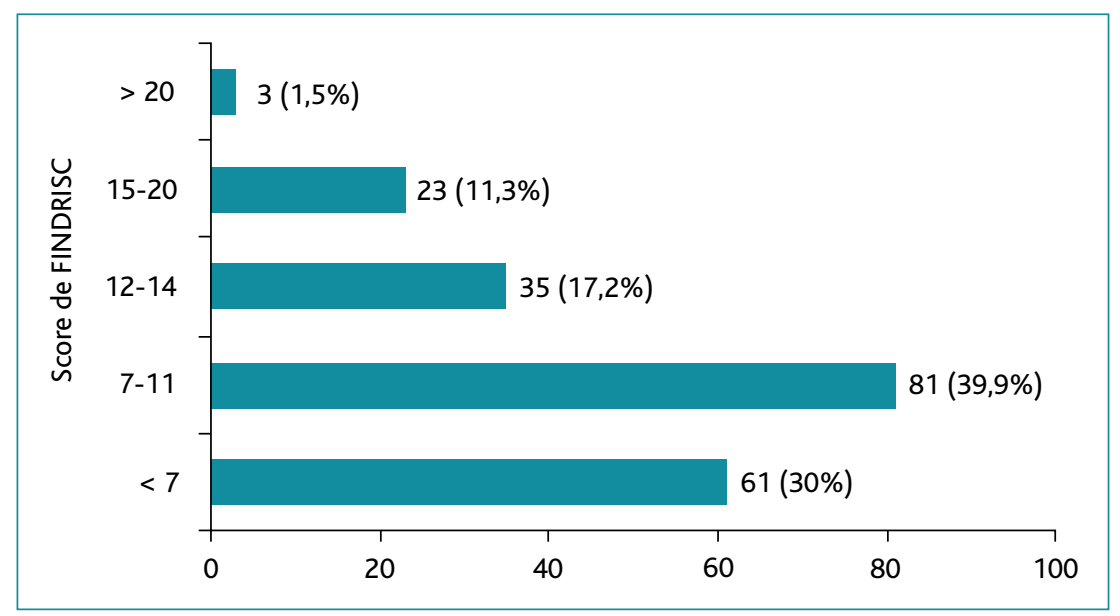

Gráfico 1. Estratificação do risco na amostra.

\section{QUADRO IV. Análise comparativa dos factores de risco por grupo FINDRISC}

\begin{tabular}{|c|c|c|c|}
\hline & $\begin{array}{l}\text { FINDRISC } \geq 15 \\
(n=26)\end{array}$ & $\begin{array}{c}\text { FINDRISC }<15 \\
(n=177)\end{array}$ & $\mathbf{P}$ \\
\hline \multicolumn{4}{|l|}{ Factores de risco não modificáveis } \\
\hline Sexo feminino (\%) & 65,4 & 61,0 & 0,669 \\
\hline Idade média (anos) & 57,7 & 43,3 & $<0,05$ \\
\hline \multicolumn{4}{|l|}{ Factores de risco modificáveis } \\
\hline IMC em Kg/m2 (média) & 31,7 & 26,1 & $<0,05$ \\
\hline Perímetro Abdominal aumentado (\%) & 88,5 & 35,6 & $<0,04$ \\
\hline HTA medicada (\%) & 73,1 & 22,0 & $<0,05$ \\
\hline Antecedentes de hiperglicemia (\%) & 42,3 & 9,0 & $<0,05$ \\
\hline Sem actividade física regular (\%) & 61,5 & 42,9 & 0,075 \\
\hline Sem ingestão regular de legumes (\%) & 23,1 & 25,4 & 0,797 \\
\hline História Familiar Diabetes $1 .^{\circ}$ grau (\%) & 80,8 & 39,5 & $<0,05$ \\
\hline FINDRISC (média) & 17,3 & 8,1 & $<0,001$ \\
\hline Homens & 17,3 & 7,48 & \\
\hline Mulheres & 17,2 & 8,52 & \\
\hline
\end{tabular}

Legenda: IMC - índice de massa corporal, H - Homens, M - Mulheres, HTA - Hipertensão Arterial petivamente, apresentaram FINDRISC $\geq 15 .{ }^{19-20}$ Contudo, salienta-se que estes estudos usaram diferentes metodologias de seleção dos participantes e a média de idades foi superior à do nosso estudo, e sendo a idade um dos fatores de risco considerados no questionário, tal pode ter contribuído também para a diferença observada. Por outro lado, as características particulares da população portuguesa poderão ter contribuído para o valor obtido, uma vez que a es- cala FINDRISC ainda não foi validada em Portugal.

Dentro do grupo de pessoas de alto risco, as médias de FINDRISC e de IMC estão próximas dos valores encontrados no estudo de prevenção primária finlandês que utilizou o score FINDRISC para identificar e referenciar os indivíduos de alto risco. ${ }^{21} \mathrm{~A}$ prevalência de excesso de peso $(46,3 \%)$ e obesidade (17,2\%) é superior à encontrada no estudo de prevalência realizado em Portugal entre 2003 e 


\section{5 (39,4\% e 14,2\%, respetivamente). ${ }^{22}$}

O presente estudo apresenta algumas limitações. O questionário FINDRISC usado para avaliar o risco dos utentes virem a desenvolver DM2 ainda não está validado para a população portuguesa. A taxa de resposta obtida foi inferior à prevista pelo que não foi possível obter a dimensão calculada da amostra. A impossibilidade de contactar alguns dos utentes da amostra constituiu o principal motivo de não participação no estudo e incluiu os que apresentavam moradas incompletas e/ou desatualizadas, números de telefone ou telemóvel errados e/ou desatualizados e aqueles que não foram contactáveis nas 3 tentativas de contacto telefónico estabelecidas no protocolo do estudo. Um dos fatores que pode ter contribuído para esta baixa taxa de resposta foi a desatualização da base de dados SINUS® do Centro de Saúde. Salienta-se ainda que o estudo foi realizado numa população de uma zona geográfica específica de Portugal pelo que não é possível generalizar os resultados à população portuguesa.

Consideram-se como pontos fortes deste estudo o uso de uma amostra aleatória de base institucional, a avaliação do risco de DM2 usando uma escala de risco internacional aplicada em inúmeros estudos e a implementação de um plano para minimização de viéses para diminuir as diferenças entre observadores na recolha de dados. Destaca-se ainda que este estudo constituiu uma oportunidade para iniciar estratégias de prevenção primária, na medida em que, os utentes que apresentaram fatores de risco de DM2 modificáveis, independentemente do risco total, foram alertados e aconselhados sobre as medidas a adotar para reduzirem o seu risco individual. Para além disso, aqueles que apresentaram FINDRISC $\geq 15$ foram sinalizados aos respetivos médicos de família, para poderem ser melhor caracterizados através da PTGO e consequentemente, desenvolvidas estratégias mais intensivas de modificação dos estilos de vida.

Por fim, este estudo sugere que um em cada oito utentes apresenta um risco elevado de desenvolver DM2 nos próximos 10 anos. Tendo em conta que um dos pilares da Medicina Geral e Familiar é a promoção da saúde e prevenção da doença, a divulgação destes resultados é importante no sentido de promover a aplicação de questionários simples e rápidos que permitam a identificação dos indivíduos com elevado risco de desenvolver DM2.

Uma vez que os recursos são limitados, a aplicação do questionário FINDRISC vai permitir selecionar os indivíduos (com risco aumentado de desenvolver DM2) que deverão ter o seu estado glicémico caracterizado, recorrendo à determinação da glicemia em jejum ou a uma PTGO, sendo que apenas esta última permitirá diagnosticar a pré-diabetes (anomalia da glicemia em jejum ou tolerância diminuída à glicose). ${ }^{3}$ Para além disso, vai permitir que a intensidade das intervenções para modificação dos estilos de vida seja ajustada ao nível de risco, assegurando que os que apresentam risco aumentado recebem intervenções mais intensivas. ${ }^{3}$

No futuro, seria interessante a realização de ensaios clínicos a longo prazo, com indivíduos de alto risco submetidos a diferentes programas de intervenção nos estilos de vida no sentido de verificar se na população portuguesa os resultados são sobreponíveis aos de outros países europeus, avaliando o custo-eficácia desses programas e procurando atingir como objetivo final e primordial - a diminuição da prevalência da diabetes no nosso país.

\section{AGRADECIMENTOS}

As autoras gostariam de agradecer a colaboração das colegas Ana Rita Novais, Eugénia Monteiro, Liliana Mota e Ana Machado na recolha dos dados para este estudo.

\section{REFERÊNCIAS BIBLIOGRÁFICAS}

1. OMS. What is diabetes? Disponivel em: http://www.who.int/mediacentre/ factsheets/fs312/en/index.html [acedido em 05/10/2011].

2. Diabetes, Factos e Números. Relatório Anual do Observatório Nacional da Diabetes. 2009. Disponível em: http://www.min-saude.pt/NR/rdonlyres/ 4747F2BE-D534-4983-9A94-C5B7066C9731/0/i012326.pdf [acedido em 05/10/2011].

3. Paulweber B, Valensi P, Lindström J, Lalic NM, Greaves CJ, McKee M, et al. A European evidence-based guideline for the prevention of type 2 diabetes. Horm Metab Res 2010 Apr; 42 Suppl 1: S3-36.

4. Wild S, Roglic G, Green A, Sicree R, King H. Global prevalence of diabetes: estimates for the year 2000 and projections for 2030. Diabetes Care 2004 May; 27 (5): 1047-53

5. Lindström J, Neumann A, Sheppard KE, Gilis-Januszewska A, Greaves CJ, Handke $\mathrm{U}$, et al. Take action to prevent diabetes - the IMAGE toolkit for the prevention of type 2 diabetes in Europe. Horm Metab Res 2010 Apr; 42 Suppl 1: S37-55.

6. Pajunen P, Landgraf R, Muylle F, Neumann A, Lindström J, Schwarz PE, et al. IMAGE Quality indicators for prevention of type 2 diabetes in Europe-IMAGE. Horm Metab Res 2010 Apr; 42 Suppl 1: 56-63.

7. Lindström J,Tuomilehto J. The diabetes risk score: a practical tool to predict type 2 diabetes risk. Diabetes Care 2003 Mar; 26 (3): 725-31.

8. Saaristo T, Peltonen M, Lindström J, Saarikoski L, Sundvall J, Eriksson JG, et al. Cross-sectional evaluation of the Finnish Diabetes Risk Score: a tool to identify undetected type 2 diabetes, abnormal glucose tolerance and metabolic syndrome. Diab Vasc Dis Res 2005 May; 2 (2): 67-72.

9. Tankova T, Chakarova N,Atanassova I, Dakovska L. Evaluation of the Finnish Diabetes Risk Score as a screening tool for impaired fasting glucose, impaired glucose tolerance and undetected diabetes. Diabetes Res Clin Pract 2011; 92 (1): 46-52.

10. Tuomilehto J, Lindström J, Eriksson JG, Valle TT, Hämäläinen H, Illane-Parikka P, et al. Prevention of type 2 diabetes mellitus by changes in lifestyle among subjects with impaired glucose tolerance. N Engl J Med 2001 May 3;344 (18): 1343 50. 
11. Knowler WC, Barrett-Connor E, Fowler SE, Hamman RF, Lachin JM, Walker EA; et al.; Diabetes Prevention Program Research Group. Reduction in the incidence of type 2 diabetes with lifestyle intervention or metformin. N Engl J Med 2002 Feb 7; 346 (6): 393-403.

12. Lindström J, Louheranta A, Mannelin M, Rastas M, Salminen V, Erikssson J, et al. The Finnish Diabetes Prevention Study (DPS): Lifestyle intervention and 3-year results on diet and physical activity. Diabetes Care 2003 Dec; 26 (12): 3230-6.

13. Lindström J, Ilanne-Parikka P, Peltonen M, Aunola S, Eriksson JG, Hemiö K, et al. Sustained reduction in the incidence of type 2 diabetes by lifestyle intervention: follow-up of the Finnish Diabetes Prevention Study. Lancet 2006 Nov 11; 368 (9548): 1673-9.

14. Costa B, Barrio F, Bolíbar B, Castell C. Prevención primaria de la diabetes tipo 2 en Cataluña mediante la intervención sobre el estilo de vida en personas de alto riesgo. Med Clin (Barc) 2007 May 12; 128 (18): 699-704.

15. Costa B, Cabré JJ, Sagarra R, Solà-Morales O, Barrio F, Piñol JL, et al. Rationale and design of the PREDICE project: cost-effectiveness of type 2 diabetes prevention among high-risk Spanish individuals following lifestyle intervention in real-life primary care setting. BMC Public Health 2011 Aug 4, 11: 623.

16. Task Force on Diabetes and Cardiovascular Diseases of the European Society of Cardiology and of the European Association for the Study of Diabetes. Guidelines on diabetes, pre-diabetes and cardiovascular diseases. Disponível em: http://www.escardio.org/guidelines-surveys/esc-guidelines/Guidelines Documents/ guidelines-diabetes-FT.pdf [acedido a 05/03/2011].

17. Direcção Geral da Saúde. Programa Nacional de Prevenção e Controlo de Diabetes. 2008. Disponível em: http://www.dgs.pt/ms/7/paginaRegisto .aspx?back=1\&id=16 474 [acedido em 05/03/2011].

18. Programa da Direção Eleita da Sociedade Portuguesa de Diabetologia. Disponível em:http://www.spd.pt/index.php?option=com_content\&task=view\&id= 56\&ltemid=29 [acedido em 05/03/2011].
19. Salinero-Fort MA, Carrillo de Santa Pau E, Abánades-Herranz JC, Dujovne-Kohan I, Cárdenas-Valladolid J; Gropu MADIABETES. Riesgo basal de diabetes mellitus en atencion primaria según cuestionario FINDRISC, factores asociados y evolución clínica tras 18 meses de seguimiento. Rev Clin Esp 2010 Oct; 210 (9): 448-53.

20. Makrilakis K, Liatis S, Grammatikou S, Perrea D, Katsilambros N. Implementation and effectiveness of the first community lifestyle intervention programme to prevent Type 2 diabetes in Greece. The DE-PLAN study. Diabet Med 2010 Apr; 27 (4): 459-65.

21. Saaristo T, Moilanen L, Jokelainen J, Korpi-Hyövälti E, Vanhala M, Saltevo J, et al. Cardiometabolic profile of people screened for high risk of type 2 diabetes in a national diabetes prevention programme (FIN-D2D). Prim Care Diabetes 2010 Dec; 4 (4): 231-9.

22. Carmo I, Santos O, Camolas J, Vieira J, Carreira M, Medina L, et al. Overweight and obesity in Portugal: national prevalence in 2003-2005. Obes Rev 2008 Jan; 9 (1): 11-19.

\section{CONFLITOS DE INTERESSE}

As autoras declaram não possuir qualquer tipo de conflitos de interesse.

\author{
ENDEREÇO PARA CORRESPONDÊNCIA \\ Teresa Valente \\ Rua da Agudela, 777, R/C direito \\ 4455-020 Lavra - Matosinhos \\ teresavalente@gmail.com
}

Recebido em 09/11/2011

Aceite para publicação em 31/01/2012

\section{ABSTRACT}

\section{RADAR STUDY - HIGH RISK FOR DIABETES IN AMARANTE}

Objectives: To determine the risk of developing diabetes mellitus type 2 (DM2) in the next ten years among patients attending a primary care clinic and to analyze the risk factors associated with developing this disease.

Type of study: Cross-sectional

Location: Amarante Primary Health Care Centre, Portugal.

Population: Patients aged 18 to 79 years old, registered with the Amarante Primary Health Care Centre ( $N=28523)$.

Methods: A random sample of 500 patients was selected for an interview. The instrument used to determine the risk for DM2 was the Finnish Diabetes Risk Score (FINDRISC) that considers eight risk factors for the disease to calculate the overall risk. The variables studied were gender and the 8 risk factors included in the FINDRISC questionnaire. Descriptive and inferential statistics were calculated.

Results: 203 patients agreed to participate. 12,8\% of subjects had an increased risk for diabetes mellitus (FINDRISC score $\geq 15$ ). Regarding the risk factors for DM2 assessed in the questionnaire, $18 \%$ of subjects had obesity (IMC $\geq 30$ ), $25 \%$ did not eat fruits and vegetables daily, $29 \%$ were taking medication for high blood pressure, $42 \%$ had waist circumference measurement criteria for central obesity, $45 \%$ had at least one member of the immediate family or other relative that had already been diagnosed with DM2, and $45 \%$ did not exercise regularly.

Discussion: This study suggests that one out of eight patients without DM2 who are treated in primary care have an increased risk of developing diabetes in the next 10 years (FINDRISC $\geq 15$ ). These results stress the importance of using a screening tool such as this scoring test. It is quick and easy to apply. It allows the family physician to perform early intervention by warning the patient of their risk and promoting healthy lifestyles.

Keywords: Diabetes Mellitus Type 2; Prevalence; Risk Assessment; Primary Health Care. 


\section{ANEXO I \\ QUESTIONÁRIO \\ (tradução publicada no Programa de Prevenção e Controlo da Diabetes)}

1. Idade anos

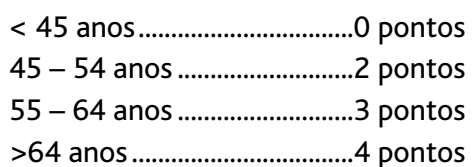

2. Índice de Massa Corporal Peso ___ kg; Altura _ _ $\mathrm{m}$

$<25$... .................................

$25-30$ . .0 pontos

$>30$ 1 ponto .3 pontos

3. Medida da cintura

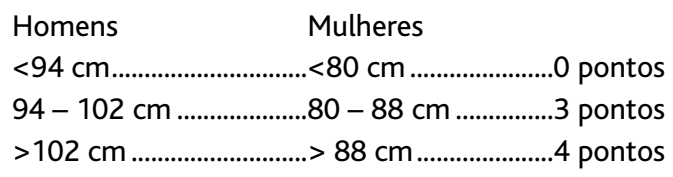

4. Pratica diariamente actividade física pelo menos $\mathbf{3 0} \mathrm{mi}-$ nutos, no trabalho ou durante o tempo livre (incluindo actividades da vida diária)?

Sim ............................................ pontos

Não .........................................2 pontos

5. Com que regularidade come vegetais e/ ou fruta?

Todos os dias 0 pontos

Às vezes. 2 pontos
6. Toma regularmente ou já tomou alguma medicação para a hipertensão arterial?

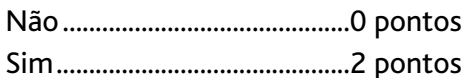

7. Alguma vez teve açúcar elevado no sangue (ex. num exame de saúde, durante um período de doença ou durante a gravidez)?

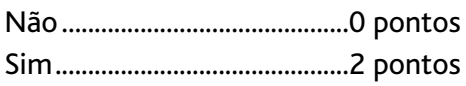

8. Tem algum membro da família próxima ou outros familiares a quem foi diagnosticado diabetes (tipo 1 ou tipo 2)? Não...................................................................... pontos

Sim: avós, tios ou primos $1 .^{\circ}$ grau .............3 pontos Sim: pais, irmãos ou filhos............................ 5 pontos

Pontuação total: pontos

Estratificação do risco:

\begin{tabular}{|cl|}
\hline Pontuação total & $\begin{array}{l}\text { Risco calculado de vir a ter diabetes tipo } \\
\text { 2 dentro de } 10 \text { anos }\end{array}$ \\
\hline$<7$ & Baixo: 1 em 100 \\
\hline $7-11$ & Discretamente elevado: 1 em 25 \\
\hline $12-14$ & Moderado: 1 em 6 \\
\hline $15-20$ & Alto: 1 em 3 \\
\hline$>20$ & Muito Alto: 1 em 2
\end{tabular}

